\title{
Suppression of Decoherence of a Spin-Boson System by Time-Periodic Control
}

\author{
Volker Bach $<$ v.bach@tu-bs.de $>$, \\ Alexander Hach < a.hach@tu-bs.de $>$, \\ Institut für Analysis und Algebra \\ TU Braunschweig \\ Pockelsstr. 14 \\ 38106 Braunschweig \\ Germany
}

19-Jan-2017

\begin{abstract}
We consider a finite-dimensional quantum system coupled to the bosonic radiation field and subject to a time-periodic control operator. Assuming the validity of a certain dynamic decoupling condition we approximate the system's time evolution with respect to the non-interacting dynamics. For sufficiently small coupling constants $g$ and control periods $T$ we show that a certain deviation of coupled and uncoupled propagator may be estimated by $\mathcal{O}(g t T)$. Our approach relies on the concept of Kato stability and general theory on non-autonomous linear evolution equations.
\end{abstract}

Keywords: Decoherence - Quantum control theory · Open quantum systems · Kato stability 


\section{Result and Discussion}

We consider an open quantum system consisting of a small, finite-dimensional system $\mathrm{S}$ coupled to a reservoir $\mathrm{R}$ with infinitely many degrees of freedom.

Specifically, we assume the small system to be an $N$-level atom, for some $N \geq 2$, i.e., the system's Hilbert space is $\mathcal{H}_{\mathrm{S}}=\mathbb{C}^{N}$, with a dynamics generated by a self-adjoint Hamiltonian matrix

$$
H_{\mathrm{S}}=\operatorname{diag}\left(E_{N-1}, E_{N-2}, \ldots, E_{1}, E_{0}\right),
$$

which we assume to be diagonal with nonnegative, nondegenerate eigenvalues $E_{N-1}>E_{N-2}>\ldots>E_{1}>E_{0} \geq 0$.

The reservoir Hilbert space $\mathcal{F}_{\mathrm{R}}=\mathcal{F}_{b}[\mathfrak{h}]$ is the boson Fock space over the square-integrable functions $\mathfrak{h}:=L^{2}\left(\mathbb{R}^{3}\right)$ on $\mathbb{R}^{3}$ and carries a three-dimensional, massless scalar quantum field - a caricature of the photon field - whose dynamics is generated by the second quantization

$$
H_{\mathrm{R}}:=d \Gamma(\omega)=\int_{\mathbb{R}^{3}} \omega(k) a_{k}^{*} a_{k} d^{3} k
$$

of (the operator of multiplication by) the photon dispersion $\omega(k):=|k|$. Here, $\left\{a_{k}, a_{k}^{*}\right\}_{k \in \mathbb{R}^{3}}$ defines the standard Fock representation of the canonical commutation relation $(\mathrm{CCR})$

$$
\left[a_{p}, a_{k}\right]=\left[a_{p}^{*}, a_{k}^{*}\right]=0, \quad\left[a_{p}, a_{k}^{*}\right]=\delta(p-k), \quad a_{k} \Omega=0,
$$

for all $k, p \in \mathbb{R}^{3}$, as an operator-valued distribution, with $\Omega \in \mathcal{F}_{\mathrm{R}}$ being the normalized vacuum vector.

The Hilbert space of the composite atom-photon system $\mathrm{S}+\mathrm{R}$ is the tensor product space $\mathcal{H}_{\mathrm{SR}}=\mathcal{H}_{\mathrm{S}} \otimes \mathcal{F}_{\mathrm{R}}$. Without interaction between these two components, the dynamics is generated by the self-adjoint Hamiltonian

$$
H_{\mathrm{SR}}^{(0)}:=H_{\mathrm{S}}+H_{\mathrm{R}}
$$

where here and henceforth we leave out trivial tensor factors whenever possible and identity, e.g., $H_{\mathrm{S}} \equiv H_{\mathrm{S}} \otimes \mathbf{1}_{\mathrm{R}}$ and $H_{\mathrm{R}} \equiv \mathbf{1}_{\mathrm{S}} \otimes H_{\mathrm{R}}$.

A dipole-type interaction $g H_{\mathrm{I}}$ couples the $N$-level atom to the large reservoir, i.e., the full, interacting dynamics is generated by the self-adjoint Hamiltonian

$$
H_{\mathrm{SR}}^{(g)}:=H_{\mathrm{SR}}^{(0)}+g H_{\mathrm{I}} .
$$

Here, $g>0$ is a small coupling constant and

$$
H_{\mathrm{I}}:=Q \otimes \phi(f) \equiv Q \phi(f)=Q\left(a^{*}(f)+a(f)\right)
$$


is the self-adjoint interaction operator specified by a self-adjoint complex $N \times N$ matrix $Q=Q^{*}$ times the field operator $\phi(f)$. Furthermore, for $f \in \mathfrak{h}$,

$$
a^{*}(f):=\int f(k) a_{k}^{*} d^{3} k, \quad a(f):=\int \overline{f(k)} a_{k} d^{3} k .
$$

We assume that $f, \omega^{-1} f \in \mathfrak{h}$ which implies the semiboundedness and selfadjointness of $H_{\mathrm{SR}}^{(g)}$ on the domain of $H_{\mathrm{SR}}^{(0)}$, for any $g>0$, since under this assumption $H_{\mathrm{I}}$ is an infinitesimal perturbation of $H_{\mathrm{SR}}^{(0)}$.

Thanks to the self-adjointness of $H_{\mathrm{SR}}^{(g)}$, the evolution operator it generates according to Schrödinger, i.e., the solution of the initial value problem $\partial_{t} U_{\mathrm{SR}}^{(g)}(t)=$ $-i H_{\mathrm{SR}}^{(g)} U_{\mathrm{SR}}^{(g)}(t), U_{\mathrm{SR}}^{(g)}(0)=\mathbf{1}$, is the strongly continuous one-parameter unitary group $t \mapsto \exp \left[-i t H_{\mathrm{SR}}^{(g)}\right]$. Given an initial state of the atom-photon system by a density matrix $\rho_{0} \in \mathcal{L}_{+}^{1}(\mathcal{H})$, i.e., a positive operator of unit trace, the state at time $t \in \mathbb{R}$ is given by

$$
\rho_{t}=\exp \left[-i t H_{\mathrm{SR}}^{(g)}\right] \rho_{0} \exp \left[i t H_{\mathrm{SR}}^{(g)}\right] .
$$

Any initial state $\rho_{0}$ eventually evolves into the ground state or the thermal equilibrium state at zero or positive temperature, respectively, as time $t \rightarrow \infty$ grows large. This phenomenon is usually refered to as return to equilibrium. As a consequence, after a sufficiently long time has elapsed, the state becomes incoherent and any information initially encoded in it is lost. A quantum computer can only process data reliably if its calculations are finished long before the loss of coherence due to the dissipative process of return to equilibrium described above sets in.

Further perturbations additionally acting on the system would typically speed up the decoherence process. If the perturbation is suitably designed, however, the opposite effect might occur and decoherence is suppressed by the perturbation, rather than enhanced.

The present paper is devoted to the question under which conditions this suppression of decoherence occurs. More specifically, we study the influence of a time-periodic perturbation represented by a control operator $H_{\mathrm{C}}(t)$ which acts on the small system $\mathrm{S}$ only. This latter restriction is a minimal requirement for a physically realistic model: $H_{\mathrm{C}}(t)$ cannot change the environment. The control operator is assumed to be a continuous family $H_{\mathrm{C}} \in C\left[\mathbb{R} ; \mathcal{B}\left(\mathcal{H}_{\mathrm{S}}\right)\right]$ of self-adjoint complex $N \times N$ matrices such that $H_{\mathrm{C}}(t+T)=H_{\mathrm{C}}(t)$, for some time period $T>0$ and all $t \in \mathbb{R}$.

Acting on the small system as an external force, the generator of the full dynamics including the control operator $H_{\mathrm{C}}(t)$ is

$$
H_{\mathrm{SRC}}^{(g)}(t):=H_{\mathrm{SR}}^{(g)}+H_{\mathrm{C}}(t)=H_{\mathrm{S}}+H_{\mathrm{R}}+H_{\mathrm{C}}(t)+g H_{\mathrm{I}}
$$


The theory of non-autonomous linear evolution equations ensures that for the corresponding time-dependent Schrödinger equation

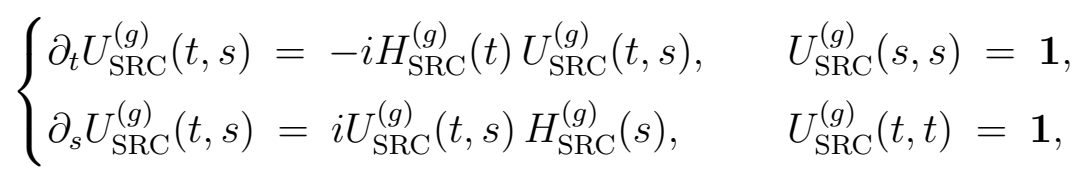

there exists a unique family $U_{\mathrm{SRC}}^{(g)} \in C^{1}\left[\Delta ; \mathcal{B}\left(\mathcal{H}_{\mathrm{SR}}\right)\right]$ of unitary operators on $\mathcal{H}_{\mathrm{SR}}$, where $\Delta:=\left\{(s, t) \in \mathbb{R}^{2} \mid s \leq t\right\} \subseteq \mathbb{R}^{2}$, that solves (10).

Our main result is Theorem 11 below which asserts that, under Decoupling Condition (14), the deviation of $U_{\mathrm{SRC}}^{(g)}(t, 0)$ from the identity is of order $\mathcal{O}(g t T)$, for times smaller than $g^{-1}$. This is to be compared to the deviation of $\exp \left[-i t H_{\mathrm{SR}}^{(g)}\right]$ from the identity which is of order $\mathcal{O}(g t)$. So, for sufficiently small time periods $T>0$, the control operator effectively slows down the evolution and hence also the decoherence of the system.

To formulate the decoupling condition, we denote by $U_{\mathrm{C}} \in C^{1}\left[\Delta ; \mathcal{B}\left(\mathcal{H}_{\mathrm{S}}\right)\right]$ the propagator generated by $H_{\mathrm{C}}(t)$, i.e., the unique solution of

$$
\begin{cases}\partial_{t} U_{\mathrm{C}}(t, s)=-i H_{\mathrm{C}}(t) U_{\mathrm{C}}(t, s), & U_{\mathrm{C}}(s, s)=\mathbf{1}, \\ \partial_{s} U_{\mathrm{C}}(t, s)=i U_{\mathrm{C}}(t, s) H_{\mathrm{C}}(s), & U_{\mathrm{C}}(t, t)=\mathbf{1},\end{cases}
$$

and $\widetilde{Q}(\tau):=U_{\mathrm{C}}(\tau, 0) Q U_{\mathrm{C}}(\tau, 0)^{*}$ on $\mathcal{H}_{\mathrm{S}}$. Our main result is as follows.

Theorem 1. Let $L \in \mathbb{N}$, assume that $\omega^{-1} f \in \mathfrak{h}$ and $\omega^{L+2} f \in \mathfrak{h}$, and set

$$
\begin{aligned}
M & :=2\left\|\left(\omega^{-1 / 2}+1\right) f\right\|_{2}+2 \sum_{k=1}^{L+2}\left(\begin{array}{c}
L+2 \\
k
\end{array}\right)\left\|\left(\omega^{k}+1\right) f\right\|_{2}, \\
C_{\mathrm{SRC}}^{(0)} & :=1+\left\|H_{\mathrm{S}}\right\|+\sup _{0 \leq r \leq T}\left\|H_{\mathrm{C}}(r)\right\| .
\end{aligned}
$$

Further assume that $g\|Q\| M T \leq 1$ and that the following decoupling condition

$$
\int_{0}^{T} \widetilde{Q}(\tau) d \tau=\int_{0}^{T} U_{\mathrm{C}}(\tau, 0) Q U_{\mathrm{C}}(\tau, 0)^{*} d \tau=0
$$

holds true. Then, for any $t \geq 0$ and with $n:=\left\lfloor\frac{t}{T}\right\rfloor, \delta:=t-n \cdot T$ as well as $\widetilde{C}_{g}:=M\|Q\| g \cdot \max \left\{1,4 C_{\mathrm{SRC}}^{(0)}+3 M\|Q\| g\right\}$,

$$
\begin{aligned}
\|\left(H_{\mathrm{R}}+\mathbf{1}\right)^{L} & \left(U_{\mathrm{SRC}}^{(g)}(t, 0)-U_{\mathrm{SRC}}^{(0)}(t, 0)\right)\left(H_{\mathrm{R}}+\mathbf{1}\right)^{-L-2} \| \\
& \leq T \cdot M\|Q\| g\left[\delta+\left(4 C_{\mathrm{SRC}}^{(0)}+3 M\|Q\| g\right) n T\right] \exp [\|Q\| M g t] \\
& \leq T \cdot \widetilde{C}_{g} t \exp [\|Q\| M g t] .
\end{aligned}
$$


We discuss Theorem 1:

- The idea of suppression of decoherence by a periodic control goes back to [5]. Theorem 1 was proven with mathematical rigor in [3], but under stronger assumptions and with considerably more involved methods:

- First, the reservoir in [3] was assumed to represent a fermion, rather than a boson field.

- Secondly, the control operator $H_{\mathrm{C}}(t)$ was assumed to commute with the Hamiltonian $H_{\mathrm{S}}$ of the atom, $\left[H_{\mathrm{C}}(t), H_{\mathrm{S}}\right]=0$, for all $t \in \mathbb{R}$.

- A third difference is the framework of Liouvilleans as generators of the dynamics at nonzero temperatures which is considerably more involved on a technical level.

- On the other hand, the approach in [3] yields control on the dynamics for all times - large and small - and, in particular, allows to follow the rate of convergence to the limiting state, as $t \rightarrow \infty$. In contrast, the methods used in the present paper give nontrivial estimates only for times less than $g^{-1}$, which is large compared to unity but small compared to the van Hove time scale $\sim g^{-2}$.

- We observe that Decoupling Condition (14) and $\partial_{t} \widetilde{Q}(t)=-i\left[H_{\mathrm{C}}(t), \widetilde{Q}(t)\right]$ imply

$$
-T \widetilde{Q}(0)=\int_{0}^{T} d t(\widetilde{Q}(t)-\widetilde{Q}(0))=-i \int_{0}^{T} d t \int_{0}^{t} d s\left[H_{\mathrm{C}}(s), \widetilde{Q}(s)\right]
$$

Since $\|\widetilde{Q}(s)\|=\|Q\|$, for all $s \in[0, T]$, the triangle inequality hence yields

$$
\int_{0}^{T}\left\|H_{\mathrm{C}}(t)\right\| d t \geq \frac{1}{2}
$$

This estimate shows that due to Decoupling Condition (14) the action the control operator excerts on the system in a single cycle is at least of the order of unity with respect to natural units $(\hbar=1)$. Assuming a control period $T$ corresponding to a physically feasible time resolution of a hypothetical control operator $H_{\mathrm{C}}(t)$, e.g. a femtosecond regime $T \sim 10^{-15} \mathrm{~s}$, the energy density in SI-units of such a device acting on an atom-sized quantum system would be about $10^{11} \mathrm{~J} / \mathrm{m}^{3}$.

In the following Section 2 we review some standard material on solutions of linear non-autonomous evolution equations on Banach spaces for which we focus 
on the special case of unitary propagators for the time-dependent Schrödinger equation. In order to apply this theory to the present model situation of a spinboson model with a time-periodic control, we then derive the necessary relative operator bounds. After these preparations, we proceed to the proof of Theorem 1 given in Section 3 . 


\section{Propagators and Kato Stability}

In this section we recall a standard set of sufficient conditions for the existence of a (unitary) propagator $(U(t, s))_{(t, s) \in \Delta}$ for the time-dependent Schrödinger equation

$$
\forall(t, s) \in \Delta: \begin{cases}\partial_{t} U(t, s)=-i H(t) U(t, s), & U(s, s)=\mathbf{1}, \\ \partial_{s} U(t, s)=i U(t, s) H(t), & U(t, t)=\mathbf{1},\end{cases}
$$

given by using the concept of Kato quasi-stability.

To define this notion we assume $(X,\|\cdot\|)$ to be a complex Banach space with a dense Banach subspace $Y \subseteq X$ whose norm $\|\cdot\|_{Y}$ can be written as $\|x\|_{Y}=\|\widehat{\Theta} x\|$ for a suitable linear, isometric bijection $\widehat{\Theta}: Y \rightarrow X$. We further assume that $\|\widehat{\Theta} x\| \geq\|x\|$, for all $x \in X$. The operator $\widehat{\Theta}$ allows us to avoid using the norm $\|\cdot\|_{Y}$ altogether.

Definition 2. Let $(X,|\cdot|)$ be a complex Banach space and $Y \subseteq X$ a dense Banach subspace. A family $G \equiv(G(t))_{t \in \mathbb{R}_{0}^{+}}$of densely defined, closed operators $G(t)$ is called Kato quasi-stable, if there exists a constant $C \geq 1$ and continuous maps $\beta_{0}, \beta_{1}: \mathbb{R}_{0}^{+} \rightarrow \mathbb{R}_{0}^{+}$such that following conditions B1, B2, and B3 are satisfied:

$B 1$ The operators $G$ define a norm-continuous family of bounded operators from $Y$ to $X$, i.e., $G \widehat{\Theta}^{-1} \in C\left[\mathbb{R}_{0}^{+}, \mathcal{B}(X)\right]$.

B2 The commutators $[\widehat{\Theta}, G(t)] \widehat{\Theta}^{-1}:=\widehat{\Theta} G(t) \widehat{\Theta}^{-1}-G(t)$ are densely defined on $X$ and extend to a continuous family of bounded operators, $[\widehat{\Theta}, G(t)] \widehat{\Theta}^{-1} \in$ $C\left[\mathbb{R}_{0}^{+}, \mathcal{B}(X)\right]$, with $\left\|[\widehat{\Theta}, G(t)] \widehat{\Theta}^{-1}\right\|_{\mathcal{B}(X)}=\beta_{1}(t)$.

B3 For all $n \in \mathbb{N}$, all $t_{1}, \ldots, t_{n} \in \mathbb{R}_{0}^{+}$, and all $\lambda_{1}>\beta_{0}\left(t_{1}\right), \ldots, \lambda_{n}>\beta_{0}\left(t_{n}\right)$, the norm estimate

$$
\left|\prod_{k=1}^{n}\left(\lambda_{k}-G\left(t_{k}\right)\right)^{-1}\right| \leq C \cdot \prod_{k=1}^{n} \frac{1}{\lambda_{k}-\beta_{0}\left(t_{k}\right)}
$$

holds true.

One of the main results of the theory on non-autonomous linear evolution equations is Theorem 3, below; see, e.g., [4, 1, 2]. A key element in the proof of Theorem 3 in [1] and in [2] is the Yosida approximation $G_{\lambda}(t):=-\lambda+\lambda^{2}[\lambda-$ $G(t)]^{-1}$, for $\lambda>\beta_{0}(t)$, which defines a family of bounded operators that strongly converge to $G(t)$, as $\lambda \rightarrow \infty$. 
Theorem 3. Let $(X,|\cdot|)$ be a complex Banach space, $Y \subseteq X$ a dense Banach subspace, and $G \equiv(G(t))_{t \in \mathbb{R}_{0}^{+}}$a Kato quasi-stable family of densely defined, closed operators, with $M \geq 1, \beta_{0}, \beta_{1}: \mathbb{R}_{0}^{+} \rightarrow \mathbb{R}_{0}^{+}$corresponding to Conditions B1, B2, and B3. Then there exists a unique solution $(U(t, s))_{(t, s) \in \Delta}$ for the non-autonomous linear evolution equation

$$
\forall(t, s) \in \Delta: \begin{cases}\partial_{t} U(t, s)=G(t) U(t, s), & U(s, s)=\mathbf{1}, \\ \partial_{s} U(t, s)=-U(t, s) G(t), & U(t, t)=\mathbf{1},\end{cases}
$$

which obeys the following norm bounds,

$$
\begin{gathered}
\|U(t, s)\| \leq C \int_{s}^{t} \beta_{0}(\tau) d \tau \\
\left\|\widehat{\Theta} U(t, s) \widehat{\Theta}^{-1}\right\| \leq C \int_{s}^{t}\left\{\beta_{0}(\tau)+C \beta_{1}(\tau)\right\} d \tau
\end{gathered}
$$

for all $(t, s) \in \Delta$.

If $X$ is specified to be a complex Hilbert space $\mathcal{H}, \mathcal{D}=\operatorname{Ran}(\widehat{\Theta}) \subseteq \mathcal{H}$, for some unbounded, self-adjoint operator $\widehat{\Theta} \geq 1$, and $G$ is a strongly continuous family $-i H \equiv(-i H(t))_{t \in \mathbb{R}_{0}^{+}}$of skew-adjoint operators on $\mathcal{H}$, then Condition B3 in Definition 2 is automatic with $C=1$ and $\beta_{0} \equiv 0$, and Theorem 3 can be strengthened to the following assertion.

Theorem 4. Let $\mathcal{H}$ be a separable complex Hilbert space, $\mathcal{D}=\operatorname{Ran}(\widehat{\Theta}) \subseteq \mathcal{H}$, for some unbounded, self-adjoint operator $\widehat{\Theta} \geq \mathbf{1}$, and $H \equiv(H(t))_{t \in \mathbb{R}_{0}^{+}} a$ strongly continuous family of self-adjoint operators $H(t)=H^{*}(t)$ on $\mathcal{H}$ such that $H \widehat{\Theta}^{-1},[\widehat{\Theta}, H(t)] \widehat{\Theta}^{-1} \in C\left[\mathbb{R}_{0}^{+}, \mathcal{B}(\mathcal{H})\right]$. Then there exists a unique propagator $(U(t, s))_{(t, s) \in \Delta}$ to the time-dependent Schrödinger equation

$$
\forall(t, s) \in \Delta: \begin{cases}\partial_{t} U(t, s)=-i H(t) U(t, s), & U(s, s)=\mathbf{1}, \\ \partial_{s} U(t, s)=i U(t, s) H(t), & U(t, t)=\mathbf{1},\end{cases}
$$

which is a family of unitary operators fulfilling the norm estimate

$$
\left\|\widehat{\Theta} U(t, s) \widehat{\Theta}^{-1}\right\| \leq \int_{s}^{t}\left\|[\widehat{\Theta}, G(\tau)] \widehat{\Theta}^{-1}\right\| d \tau
$$

for all $(t, s) \in \Delta$. 
To apply Theorem 4 to the present model situation, we choose

$$
\mathcal{H}:=\mathcal{H}_{\mathrm{SR}}, \widehat{\Theta}:=\Theta^{L+2}, \Theta:=H_{\mathrm{R}}+1, H(t):=H_{\mathrm{SRC}}(t) .
$$

To validate the hypothesis of Theorem 4, we define

$$
\begin{aligned}
M_{-1 / 2} & :=2\left\|\left(\omega^{-1 / 2}+1\right) f\right\|_{2}, \\
M_{n} & :=2 \sum_{k=1}^{n}\left(\begin{array}{l}
n \\
k
\end{array}\right)\left\|\left(\omega^{k}+1\right) f\right\|_{2}
\end{aligned}
$$

and establish the following bounds.

Lemma 5. Let $n \in \mathbb{N}$ and assume that $\omega^{-1 / 2} f, \omega^{n} f \in L^{2}\left(\mathbb{R}^{3}\right)$. Then

$$
\begin{aligned}
\left\|a(f) \Theta^{-1}\right\|,\left\|a^{*}(f) \Theta^{-1}\right\| & \leq \frac{1}{2} M_{-1 / 2} . \\
\left\|\left[\Theta^{n}, a^{*}(f)\right] \Theta^{-n}\right\|,\left\|\left[\Theta^{n}, a(f)\right] \Theta^{-n}\right\| & \leq \frac{1}{2} M_{n} .
\end{aligned}
$$

Proof. It is convenient to introduce the subspace $\mathcal{F}_{\mathrm{R}}^{\text {fin }} \subseteq \mathcal{F}_{\mathrm{R}}$ of finite vectors whose elements have only finitely many non-vanishing components, each being smooth and compactly supported. For any normalized finite vector $\psi \in \mathcal{F}_{\mathrm{R}}^{\text {fin }}$, we have that

$$
\left\|a^{*}\left(\omega^{k} f\right) \psi\right\|^{2}=\left\|\omega^{k} f\right\|_{2}^{2}+\left\|a\left(\omega^{k} f\right) \psi\right\|^{2},
$$

for all $k \geq 0$. Additionally requiring that $k \geq 1$, we further have

$$
\begin{aligned}
\left\|a\left(\omega^{k} f\right) \psi\right\| & \leq \int|f(\xi)|\left\|\omega^{k}(\xi) a_{\xi} \psi\right\| d \xi \leq\|f\|_{2} \cdot\left\langle\psi \mid d \Gamma\left[\omega^{2 k}\right] \psi\right\rangle^{1 / 2} \\
& \leq\|f\|_{2} \cdot\left\langle\psi \mid(d \Gamma[\omega])^{2 k} \psi\right\rangle^{1 / 2}=\|f\|_{2} \cdot\left\|H_{\mathrm{R}}^{k} \psi\right\| \leq\|f\|_{2} \cdot\left\|\Theta^{k} \psi\right\|,
\end{aligned}
$$

where $d \Gamma(A)$ denotes the second quantization of an operator $A$. For $k=0$, we slightly modify this estimate and obtain

$$
\|a(f) \psi\| \leq \int|f(\xi)|\left\|a_{\xi} \psi\right\| d \xi \leq\left\|\omega^{-1 / 2} f\right\|_{2} \cdot\left\|H_{\mathrm{R}}^{1 / 2} \psi\right\|
$$

This estimate and (30) with $k=0$ establish

$$
\left\|a(f) \Theta^{-1}\right\|,\left\|a^{*}(f) \Theta^{-1}\right\| \leq\left\|\left(\omega^{-1 / 2}+1\right) f\right\|_{2}
$$

and hence (28). 
On the other hand, Eq. (30) and (31) imply for $k \geq 1$ that

$$
\left\|a\left(\omega^{k} f\right) \Theta^{-k}\right\|,\left\|a^{*}\left(\omega^{k} f\right) \Theta^{-k}\right\| \leq\left\|\left(\omega^{k}+1\right) f\right\|_{2} .
$$

Using the identities

$$
\left[H_{\mathrm{R}}, a^{*}(f)\right]=a^{*}(\omega f), \quad\left[H_{\mathrm{R}}, a(f)\right]=-a(\omega f),
$$

and an induction, we easily find that

$$
\begin{aligned}
\Theta^{n} a^{*}(f) \Theta^{-n} & =\Theta^{n-1} a^{*}(f) \Theta^{-(n-1)}+\Theta^{n-1} a^{*}(\omega f) \Theta^{-n}=\ldots \\
& =\sum_{k=0}^{n}\left(\begin{array}{l}
n \\
k
\end{array}\right) a^{*}\left(\omega^{k} f\right) \Theta^{-k},
\end{aligned}
$$

and similarly

$$
\Theta^{n} a(f) \Theta^{-n}=\sum_{k=0}^{n}(-1)^{k}\left(\begin{array}{l}
n \\
k
\end{array}\right) a\left(\omega^{k} f\right) \Theta^{-k} .
$$

Putting (36), (37) and (34) together, we obtain

$$
\left\|\left[\Theta^{n}, a^{*}(f)\right] \Theta^{-n}\right\|,\left\|\left[\Theta^{n}, a(f)\right] \Theta^{-n}\right\| \leq \sum_{k=1}^{n}\left(\begin{array}{l}
n \\
k
\end{array}\right)\left\|\left(\omega^{k}+1\right) f\right\|_{2} .
$$

Since $\tau \mapsto H_{\mathrm{C}}(\tau)$ is continuous and $\left[H_{\mathrm{SRC}}(\tau), \Theta\right]=g Q \otimes\left[a^{*}(f)+a(f), H_{\mathrm{R}}\right]$, Lemma 5 and Theorem 4 imply the following corollary.

Corollary 6. Let $L \in \mathbb{N}_{0}$ and assume that $\omega^{-1 / 2} f, \omega^{L+2} f \in L^{2}\left(\mathbb{R}^{3}\right)$. Then $\tau \mapsto$ $H_{\mathrm{SRC}}^{(g)}(\tau) \Theta^{-1}$ is continuous and bounded, uniformly in $\tau \in \mathbb{R}$, and fulfills the following estimates,

$$
\begin{aligned}
& \left\|\Theta^{\ell} H_{\mathrm{I}} \Theta^{-(\ell+j)}\right\| \leq\|Q\|\left(M_{-1 / 2}+M_{\ell+j}\right), \\
& \left\|\left[\Theta^{\ell+j}, H_{\mathrm{SRC}}^{(g)}(\tau)\right] \Theta^{-(\ell+j)}\right\| \leq g\|Q\| M_{\ell+j},
\end{aligned}
$$

for all $\tau \in \mathbb{R}$, all $\ell \in\{0,1, \ldots, L\}$, and $j \in\{1,2\}$. Moreover, $\tau \mapsto H_{\mathrm{SRC}}^{(g)}(\tau)$ is a Kato-quasistable family of self-adjoint operators, and the unique, unitary solution of

$$
\begin{cases}\partial_{t} U_{\mathrm{SRC}}^{(g)}(t, s)=-i H_{\mathrm{SRC}}^{(g)}(t) U_{\mathrm{SRC}}^{(g)}(t, s), & U_{\mathrm{SRC}}^{(g)}(s, s)=\mathbf{1} \\ \partial_{s} U_{\mathrm{SRC}}^{(g)}(t, s)=i U_{\mathrm{SRC}}^{(g)}(t, s) H_{\mathrm{SRC}}^{(g)}(s), & U_{\mathrm{SRC}}^{(g)}(s, s)=\mathbf{1}\end{cases}
$$

obeys

$$
\left\|\Theta^{\ell+j} U_{\mathrm{SRC}}^{(g)}(t, s) \Theta^{-(\ell+j)}\right\| \leq \exp \left[g\|Q\| M_{\ell+j}(t-s)\right],
$$

for all $(t, s) \in \Delta$, all $\ell \in\{0,1, \ldots, L\}$, and $j \in\{1,2\}$. 


\section{Proof of Theorem 1}

We first fix $n \in \mathbb{N}$ and $\delta \in[0,1)$ so that $t=n T+\delta$. We abbreviate

$$
\Theta:=H_{\mathrm{R}}+\mathbf{1}, U_{\kappa}(s):=U_{\mathrm{SRC}}^{(\kappa)}(s, 0), W(s):=U_{0}(s)^{*} U_{g}(s)-\mathbf{1},
$$

for $\kappa \geq 0$. Next we claim that, for all $s \geq 0$,

$$
U_{0}(s+T)=U_{0}(s) U_{0}(T), \quad U_{g}(s+T)=U_{g}(s) U_{g}(T) .
$$

Indeed, $\partial_{s} U_{\kappa}(s) U_{\kappa}(T)=-i H_{\mathrm{SRC}}^{(\kappa)}(s) U_{\kappa}(s) U_{\kappa}(T)$ and

$$
\partial_{s} U_{\kappa}(s+T)=-i H_{\mathrm{SRC}}^{(\kappa)}(s+T) U_{\kappa}(s+T)=-i H_{\mathrm{SRC}}^{(\kappa)}(s) U_{\kappa}(s+T),
$$

since $H_{\mathrm{SRC}}^{(\kappa)}$ is $T$-periodic. The uniqueness of the solution of linear ODE with the same initial value then implies (44) both for $\kappa=0$ and for $\kappa=g$.

Eq. (44) in turn implies that

$$
\begin{aligned}
U_{g}(t)-U_{0}(t)= & U_{g}(\delta+n T)-U_{0}(\delta+n T) \\
= & {\left[U_{g}(\delta)-U_{0}(\delta)\right] U_{g}(n T)+U_{0}(\delta)\left[U_{g}(n T)-U_{0}(n T)\right] } \\
= & U_{0}(\delta) W(\delta) U_{g}(n T) \\
& \quad+\sum_{j=1}^{n} U_{0}(\delta+(j-1) T)\left[U_{g}(T)-U_{0}(T)\right] U_{g}((n-j) T) \\
= & U_{0}(\delta) W(\delta) U_{g}(n T)+\sum_{k=0}^{n-1} U_{0}(\delta+(n-k) T) W(T) U_{g}(k T) .
\end{aligned}
$$

Since $U_{0}(s)$ is unitary and commutes with $\Theta$ this identity implies that

$$
\begin{aligned}
& \| \Theta^{L}\left(U_{g}(t)\right.\left.-U_{0}(t)\right) \Theta^{-L-2} \| \\
& \leq\left\|\Theta^{L} W(\delta) U_{g}(n T) \Theta^{-L-2}\right\|+\sum_{k=0}^{n-1}\left\|\Theta^{L} W(T) U_{g}(k T) \Theta^{-L-2}\right\| \\
& \leq\left(\left\|\Theta^{L} W(\delta) \Theta^{-L-2}\right\|+n \cdot\left\|\Theta^{L} W(T) \Theta^{-L-2}\right\|\right) \\
& \cdot \sup _{0 \leq \tau \leq n T}\left\|\Theta^{L+2} U_{g}(\tau) \Theta^{-L-2}\right\|
\end{aligned}
$$

Thanks to Corollary 6, Eq. (42) above, we have that

$$
\left\|\Theta^{L+2} U_{g}(\tau) \Theta^{-L-2}\right\| \leq \exp [\|Q\| M g \tau]
$$


for all $\tau \geq 0$, using that $M_{L+2} \leq M$. Furthermore, for all $0 \leq s \leq T$, the fundamental theorem of calculus gives

$$
\begin{aligned}
\Theta^{L+1} W(s) \Theta^{-L-2} & =i \int_{0}^{s} \Theta^{L+1} U_{0}(s)^{*}\left(H_{\mathrm{SRC}}^{(0)}(t)-H_{\mathrm{SRC}}^{(g)}(t)\right) U_{g}(s) \Theta^{-L-2} d s \\
& =i g \int_{0}^{s} \Theta^{L+1} U_{0}(s)^{*} H_{\mathrm{I}} U_{g}(s) \Theta^{-L-2} d s
\end{aligned}
$$

and with (48) and Corollary 6, Eq. (39) this implies that

$$
\begin{aligned}
\left\|\Theta^{L} W(\delta) \Theta^{-L-2}\right\| & \leq\left\|\Theta^{L+1} W(\delta) \Theta^{-L-2}\right\| \\
& \leq g \delta\left\|\Theta^{L+1} H_{\mathrm{I}} \Theta^{-L-2}\right\| \cdot \exp [\|Q\| M g \delta] \\
& \leq g \delta\|Q\| M \exp [\|Q\| M g \delta],
\end{aligned}
$$

additionally using that $M=M_{-1 / 2}+M_{L+2}$. Inserting (48) and (50) into (47), we obtain

$$
\begin{aligned}
& \left\|\Theta^{L}\left(U_{g}(t)-U_{0}(t)\right) \Theta^{-L-2}\right\| \\
& \quad \leq\left(g \delta\|Q\| M+n \cdot\left\|\Theta^{L} W(T) \Theta^{-L-2}\right\|\right) \cdot \exp [\|Q\| M g t] .
\end{aligned}
$$

For the estimate of $\left\|\Theta^{L} W(T) \Theta^{-L-2}\right\|$, we observe that, again by the fundamental theorem of calculus,

$$
\begin{aligned}
\Theta^{L} W(T) \Theta^{-L-2} & =i g \int_{0}^{T} \Theta^{L} U_{0}(s)^{*} H_{\mathrm{I}} U_{g}(s) \Theta^{-L-2} d s \\
= & i g \int_{0}^{T} \Theta^{L} U_{0}(s)^{*} H_{\mathrm{I}} U_{0}(s) W(s) \Theta^{-L-2} d s \\
& +i g \int_{0}^{T} \Theta^{L} U_{0}(s)^{*} H_{\mathrm{I}} U_{0}(s) \Theta^{-L-2} d s
\end{aligned}
$$

which implies that

$$
\begin{aligned}
\left\|\Theta^{L} W(T) \Theta^{-L-2}\right\| \leq & g\left\|\Theta^{L} H_{\mathrm{I}} \Theta^{-L-1}\right\| \int_{0}^{T}\left\|\Theta^{L+1} W(s) \Theta^{-L-2}\right\| d s \\
& +g\left\|\int_{0}^{T} \Theta^{L} U_{0}(s)^{*} H_{\mathrm{I}} U_{0}(s) \Theta^{-L-2} d s\right\| \\
\leq & g^{2} T^{2}\|Q\|^{2} M^{2} \exp [\|Q\| M g T] \\
& +g\left\|\int_{0}^{T} \Theta^{L} U_{0}(s)^{*} H_{\mathrm{I}} U_{0}(s) \Theta^{-L-2} d s\right\|
\end{aligned}
$$


using (50) and again (39) and $M=M_{-1 / 2}+M_{L+2}$.

We proceed to the key estimate of this paper whose proof uses Decoupling Condition (14). Namely, we observe that

$$
\begin{aligned}
& \int_{0}^{T} U_{0}(s)^{*} H_{\mathrm{I}} U_{0}(s) d s=\int_{0}^{T} d s\left\{U_{0}(s)^{*} H_{\mathrm{I}} U_{0}(s)-U_{\mathrm{C}}(s)^{*} H_{\mathrm{I}} U_{\mathrm{C}}(s)\right\} \\
& =\int_{0}^{T} d s \int_{0}^{s} d r\left\{U_{0}(r)^{*}\left[H_{\mathrm{SRC}}^{(0)}(r), H_{\mathrm{I}}\right] U_{0}(r)-U_{\mathrm{C}}(r)^{*}\left[H_{\mathrm{C}}(r), H_{\mathrm{I}}\right] U_{\mathrm{C}}(r)\right\} .
\end{aligned}
$$

Since $U_{0}(r), U_{\mathrm{C}}(r), H_{\mathrm{S}}, H_{\mathrm{R}}$, and $H_{\mathrm{C}}(r)$ all commute with $\Theta$, this, (39), and $M=M_{-1 / 2}+M_{L+2}$ imply that

$$
\begin{gathered}
\left\|\int_{0}^{T} \Theta^{L} U_{0}(s)^{*} H_{\mathrm{I}} U_{0}(s) \Theta^{-L-2} d s\right\| \\
\leq 2 T^{2}\left(\left\|\Theta^{L} H_{\mathrm{I}} \Theta^{-L-1}\right\|+\left\|\Theta^{L+1} H_{\mathrm{I}} \Theta^{-L-2}\right\|\right) \\
\cdot\left(\left\|H_{\mathrm{R}} \Theta^{-1}\right\|+\left\|H_{\mathrm{S}}\right\|+\sup _{0 \leq r \leq T}\left\|H_{\mathrm{C}}(r)\right\|\right) \\
\leq 4 C_{\mathrm{SRC}}^{(0)} T^{2}\|Q\| M,
\end{gathered}
$$

where $C_{\mathrm{SRC}}^{(0)}:=1+\left\|H_{\mathrm{S}}\right\|+\sup _{0 \leq r \leq T}\left\|H_{\mathrm{C}}(r)\right\|$. Inserting (55) into (53) and the resulting estimate into (51), we arrive at the assertion, taking into account that $g\|Q\| M T \leq 1$ which implies that $\exp [\|Q\| M g T] \leq e \leq 3$.

\section{References}

[1] V. Bach and J.-B. Bru. Rigorous foundations of the brockett-wegner flow for operators. J. Evol. Equ., 10:425-442, 2010.

[2] V. Bach and J.-B. Bru. Diagonalizing quadratic bosonic operators by nonautonomous flow equation. Memoirs of the AMS, 240(1138):1-122, Mar 2016.

[3] V. Bach, W. de Siquiera Pedra, M. Merkli, and I. M. Sigal. Suppression of decoherence by periodic forcing. J. Stat. Phys, 155(6):1271-1298, Jun 2014.

[4] K.-J. Engel and R. Nagel. A Short Course on Operator Semigroups. Universitext. Springer-Verlag, 2006. 
[5] P. Facchi, S. Tasaki, S. Pascazio, H. Nakazato, A. Tokuse, and D. A. Lidar. Control of decoherence: Analysis and comparison of three different strategies. Phys. Rev. A, 71(2):022302, Feb 2005. 